\title{
Carbono acumulado en la biomasa aérea en plantaciones de Terminalia ivorensis A. Chev (terminalia) y Gmelina arborea Roxb (melina), en el Litoral ecuatoriano
}

\section{Accumulated carbon in the aerial biomass of Terminalia ivorensis A. Chev (terminalia) y Gmelina arborea Roxb (melina) plantation, in the Ecuadorian Coast}

\author{
Betty González Osorio ${ }^{1}$, Pedro Suatunce Cunuhay ${ }^{1}$, Luís Simba Ochoa ${ }^{1}$. \\ ${ }^{1}$ Universidad Técnica Estatal de Quevedo, Facultad de Ciencias Ambientales, Carrera de Ingeniería Forestal. Campus "Ing. \\ Manuel Haz Álvarez”, Av. Quito, km 1.5 vía a Santo Domingo de los Tsáchilas. EC.120301. Quevedo, Ecuador: \\ bgonzalez@uteq.edu.ec
}

Rec.: 17.08.2018. Acept.: 10.12.2018.

Publicado el 30 de junio de 2019

\section{Resumen}

$\mathrm{E}_{\mathrm{s}}^{\mathrm{s}}$ sta investigación se realizó con el objetivo de cuantificar el carbono (C) almacenado en la biomasa aérea de plantaciones de Gmelina arborea (melina) y Terminalia ivorensis (terminalia), de nueve años de edad, en tres localidades del Litoral ecuatoriano, mediante la utilización de ecuaciones alométricas. En cada localidad se ubicaron, al azar, cuatro áreas de muestreo, para cada especie, y en cada área de muestreo se establecieron cuatro parcelas de $500 \mathrm{~m}^{2}$, en los cuales se registró el DAP (diámetro a la altura de pecho $=1.30$ m sobre el suelo) y la altura total (h). Para estimar la biomasa aérea se utilizó la ecuación alométrica $\mathrm{Bt}=$ $5.025173+0.021553 * \mathrm{DAP}^{2} \mathrm{~h}$ ) y para estimar el carbono se multiplicó la biomasa aérea $(\mathrm{Bt})$ por 0.5 , según la guía del IPCC. El potencial de la fijación de carbono se determinó mediante el incremento medio anual (IMA) del volumen. Se encontraron diferencias significativas, entre las especies y entre las localidades, para la biomasa, carbono almacenado y fijado, en la parte aérea de las plantaciones. El promedio de la biomasa aérea, carbono almacenado y fijado fue mayor para la especie $G$. arborea; la localidad con mayor promedio de biomasa, carbono almacenado y fijado fue Santo Domingo, y la localidad con menor promedio fue Balzar.

Palabras clave: biomasa aérea, carbono, especies forestales, DAP, altura total.

\begin{abstract}
$\mathrm{T}$ his research was carried out with the objective of quantifying the carbon (C) stored in the air biomass of Gmelina arborea (melina) and Terminalia ivorensis (terminalia) plantations, of nine years of age, in three localities of the ecuadorian Coast, by the use of allometric equations. In each locality four sampling areas, at random, were located for each species, and in each sampling area four parcels of $500 \mathrm{~m}^{2}$ was settled down, in which the DBH (diameter at breast height $=1.30 \mathrm{~m}$ on the floor) and the total height (h) was registered. To estimate the air biomass the allometric equation $\mathrm{Bt}=5.025173+0.021553 * \mathrm{DAP}^{2} \mathrm{~h}$ ) was used, and to estimate the carbon it was multiplied the air biomass (Bt) for 0.5 , according to the IPCC guide. The potential of the fixation of carbon was determined by the half annual increment (IMA) of the volume. There were significant differences, among the species and among the locality, for the biomass, stored and fixed carbon, in the air part of the plantations. The average of the aerial biomass stored, and fixed carbon was bigger for the species $G$. arborea; the locality with more average of biomass, stored and fixed carbon was Santo Domingo, and the town with smaller average was Balzar.
\end{abstract}

Key words: aerial biomass, carbon, forest species, DBH, total height. 


\section{Introducción}

$\mathrm{E}$ lincremento de la temperatura atmosférica, causado por el incremento de la concentración de los gases de efecto invernadero en la atmosfera, es una amenaza mundial para la humanidad, con efectos negativos en los ámbitos biológicos, económicos y sociales (Fonseca et al., 2008). La mayoría de las estrategias de mitigación del efecto invernadero tienen como objetivo la reducción de la concentración de carbono en la atmosfera, el principal gas de infecto invernadero. Las plantaciones forestales constituyen los principales sumideros de carbono y contribuyen a la mitigación del cambio climático, ya que los ecosistemas forestales contienen grandes cantidades de carbono almacenado en la biomasa aérea viva y muerta, así como en el suelo. Las plantaciones forestales son capaces de remover el carbono de la atmósfera por medio de la fotosíntesis y almacenar en la biomasa aérea y en el suelo (Rojas, 2014).

La terminalia o roble marfil (Terminalia ivorensis A. Chev.) es una especie forestal originaria de las regiones tropicales de África Occidental que, en su hábitat natural, se encuentra en estado de vulnerabilidad, debido a su escasa regeneración natural (IUCN, 2018). La terminalia es una especie de rápido crecimiento, que puede alcanzar alturas superiores a $45 \mathrm{~m}$ y DAP mayores a $60 \mathrm{~cm}$. Por su buena adaptación y variedad de usos de su madera, en los últimos años, esta especie se está utilizando en plantaciones forestales de Centro y Sur América (Lamb y Ntima, 1971; Montes, 2017).

La melina o gemelina (Gmelina arborea Roxb.) es una especie forestal de muy rápido crecimiento, que alcanza los $30 \mathrm{~m}$ de altura y DAP superiores a $80 \mathrm{~cm}$. Esta especie es originaria de las regiones tropicales y subtropicales de Asia, que se utiliza en los proyectos de reforestación en América Central y Sur América, por su rápido crecimiento, facilidad de manejo y los múltiples usos de su madera (Rojas et al., 2004).

El mantenimiento de reservas de carbono en plantaciones forestales se ha convertido en un servicio ambiental reconocido a escala global, el mismo que tiene un valor considerable para los países en vías de desarrollo. Para esto es necesario cuantificar la cantidad de biomasa y el contenido de carbono en las plantaciones forestales (Derek et al., 2000; Snowdon et al., 2001). Para estimar la cantidad de carbono que se logra eliminar de la atmósfera, mediante el establecimiento de plantaciones forestales, es necesario contar con procedimientos apropiados, como los modelos para cuantificar la biomasa de los árboles (Montero y Kanninen, 2003). Este proyecto pretende determinar el carbono almacenado en la biomasa aérea de plantaciones de T. ivorensis y G. arborea, en tres localidades del Litoral ecuatoriano, mediante la aplicación de modelos alométricos.

\section{Materiales y métodos}

\section{Localización del área de estudio}

Esta investigación se realizó en tres haciendas de la empresa SERAGROFOREST S.A., ubicadas en los cantones: Balzar(Guayas), que se encuentra a una altitud de $40 \mathrm{msnm}$, entre las coordenadas geográficas $01^{\circ} 21^{\prime}$ $39^{\prime \prime}$ de latitud sur y $79^{\circ} 04^{\prime} 00^{\prime \prime}$, con una temperatura promedio de $25.6^{\circ} \mathrm{C}$, precipitación anual de $1095 \mathrm{~mm}$, corresponde a la zona de vida bosque seco Tropical (bsT). Santo Domingo de Los Colorados (Santo Domingo), que se encuentra a una altitud $625 \mathrm{msnm}$, entre las coordenadas geográficas $0^{\circ} 15 ' 10.98$ " de latitud sur y $79^{\circ} 10^{\prime} 31.3^{\prime \prime}$ longitud oeste, con una temperatura media de $23^{\circ} \mathrm{C}$, precipitación de $3150 \mathrm{~mm}$, corresponde a la zona de vida bosque húmedo Subtropical (bh-S). Valencia (Los Ríos), que se encuentra ubicado a una altitud de $80 \mathrm{msnm}$, entre las coordenadas geográficas 0॰57'09" latitud sur y 79²1'11" longitud oeste, con una temperatura media de $25^{\circ} \mathrm{C}$, precipitación anual de $2162 \mathrm{~mm}$, corresponde a la zona de vida bosque húmedo Subtropical (bh-S) (Ecured, 2018; Clima Data, 2018) (Figura 1).

\section{Establecimiento de las parcelas de muestreo}

En cada localidad se seleccionaron, al azar, cuatro áreas de muestreo dentro de la plantación de T. ivorensis y $G$. arborea, de nueve años de edad. En cada área de muestreo se establecieron cuatro parcelas de muestreo rectangulares de $500 \mathrm{~m}^{2}$ ( $\left.25 \mathrm{~m} \times 20 \mathrm{~m}\right)$. con un total de 16 unidades de muestreo para especie en cada localidad. En cada parcela de muestreo se registró los datos de DAP (diámetro a $1.30 \mathrm{~m}$ sobre el suelo) y altura total de todos los árboles. Además, se determinó el número de árboles por hectárea $(\mathrm{N})$ mediante la fórmula:

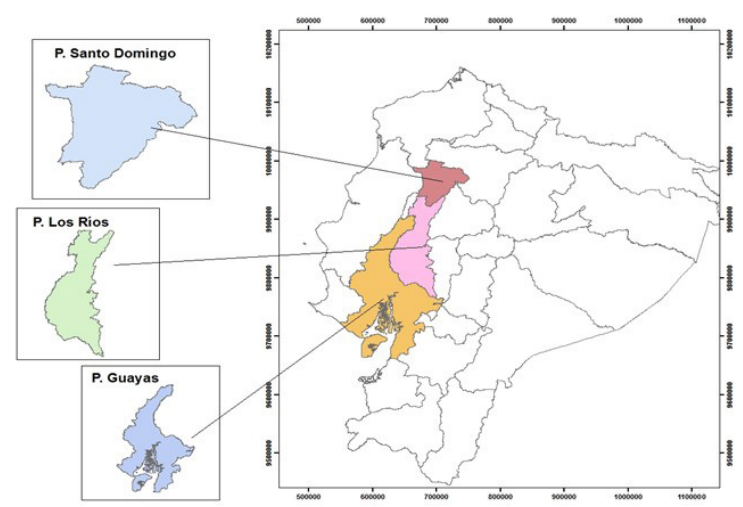

Figura 1. Ubicación de las provincias donde se seleccionaron las parcelas de muestreo. 


$$
\mathrm{N}=\frac{(n * 1 h a)}{0.05 h a}
$$

Donde:

$\mathrm{N}$ = número de árboles por hectárea

$\mathrm{n}=$ número de árboles de la parcela

ha $=$ hectárea

\section{Estimación de la biomasa y carbono almacenado}

Para estimar la biomasa y el carbono almacenados en la parte aérea de los árboles de $T$. ivorensis y $G$. arborea, primero se procedió a calcular la biomasa total (Bt), de la parte aérea, de cada especie por medio de la ecuación propuesta por Loguercio y Defossé (2001), como se indica:

$$
\mathrm{Bt}=5.025173+0.021553 * \mathrm{DAP}^{2} \mathrm{~h}
$$

Donde:

$\mathrm{Bt}=$ biomasa total aérea $(\mathrm{kg})$

$\mathrm{DAP}=$ diámetro a $1.30 \mathrm{~m}$ sobre el suelo $(\mathrm{cm})$

$\mathrm{h}=$ altura total del árbol $(\mathrm{m})$

El carbono de la biomasa aérea se obtuvo multiplicando la biomasa aérea $(\mathrm{Bt})$ obtenida por 0.5 , según la guía establecida por la IPCC (2006).

Incremento medio anual (IMA) del volumen y fijación de carbono

Para determinar el incremento medio anual, primero se calculó el volumen por árbol, mediante la ecuación siguiente:

$\mathrm{v}=\pi / 4 * \mathrm{DAP}^{2 *} \mathrm{~h} * \mathrm{f}$

Donde:

$\mathrm{v}=$ volumen del árbol $\left(\mathrm{m}^{3}\right)$ $\pi=$ constante $=3.1416$

DAP $=$ diámetro a $1.30 \mathrm{~m}$ sobre el suelo $(\mathrm{cm})$

$\mathrm{h}=$ altura total del árbol $(\mathrm{m})$

$\mathrm{f}=$ factor de forma $=0.6$

El valor del volumen obtenido se dividió para los nueve años de edad y se obtuvo el IMA por árbol. Este resultado se multiplicó por la densidad y se obtuvo el IMA del volumen por hectárea. Con el IMA calculado se proyectó el volumen hasta la edad de 12 años para las dos especies forestales. El potencial de la fijación del carbono fue estimado mediante el volumen esperado a la edad de 12 años, en las dos especies forestales.

\section{Análisis estadísticos}

Se realizó un análisis de varianza combinado para determinar las diferencias de la biomasa aérea, carbono almacenado y fijado, entre especies y entre localidades. Para la comparación de promedios se aplicó la prueba de rangos múltiples de Tukey, al 5\% de probabilidad de error, utilizando un software libre.

\section{Resultados y discusión}

\section{Biomasa aérea de las especies forestales}

El promedio de la biomasa total, de la parte aérea, presentó diferencias significativas $(\mathrm{p}<0.05)$ entre las especies y entre las localidades. La mayor biomasa total fue para la especie G. arborea; la localidad con mayor biomasa total fue Santo Domingo y la localidad con menor biomasa total fue Balzar (Cuadro 1).

Los promedios de la biomasa total registrado en las tres localidades para G. arborea, a los nueve años de edad, fueron superiores a las $64.84 \mathrm{t} / \mathrm{ha}^{-1}$ obtenidas por Melo (2015), en plantaciones de G. arborea de diez años de edad. Los promedios de biomasa total registrados en las tres localidades para $T$. ivorensis, a los nueve años de edad, fueron superiores a los $24.8 \mathrm{t} / \mathrm{ha}^{-1}$ reportados

Cuadro 1. Biomasa (t ha $\left.{ }^{-1}\right)$ de G. arborea y $T$. ivorensis en tres localidades del Litoral ecuatoriano

\begin{tabular}{lcccc}
\hline \multirow{2}{*}{ Especies } & \multicolumn{3}{c}{ Localidades } & Promedio \\
\cline { 2 - 4 } & Sto. Domingo & Valencia & Balzar & \\
\hline Gemelina arborea & 227.18 & 190.39 & 190.957 & $202.84 \mathrm{a}$ \\
Terminalia ivorensis & 30.98 & 50.79 & 30.28 & $37.35 \mathrm{~b}$ \\
Promedio & $129.08 \mathrm{a}$ & $120.59 \mathrm{ab}$ & $110.62 \mathrm{~b}$ & 120.10 \\
& & & \\
\hline $\mathrm{CV}(\%)$ & & & 29.81 \\
\hline
\end{tabular}


González et al., 2019

Cuadro 2. Carbono $\left(\mathrm{t} \mathrm{ha}^{-1}\right)$ de G. arborea y $T$. ivorensis en tres localidades del Litoral ecuatoriano

\begin{tabular}{lcccc}
\hline \multirow{2}{*}{ Especies } & \multicolumn{3}{c}{ Localidades } & Promedio \\
\cline { 2 - 4 } & Sto. Domingo & Valencia & Balzar & \\
\hline Gemelina arborea & 113.587 & 95.197 & 95.478 & $101.42 \mathrm{a}$ \\
Terminalia ivorensis & 15.495 & 25.396 & 15.138 & $18.68 \mathrm{~b}$ \\
Promedio & $64.54 \mathrm{a}$ & $60.30 \mathrm{ab}$ & $55.31 \mathrm{~b}$ & 120.10 \\
& & & & 28.76 \\
\hline $\mathrm{CV}(\%)$
\end{tabular}

por (Magaña et al., 2004) en sistemas agroforestales con café y $T$. ivorensis, a los siete años de edad, en Costa Rica.

Carbono almacenado en la biomasa aérea de las especies forestales

El promedio del carbono almacenado en la biomasa aérea presentó diferencias significativas $(\mathrm{p}<0.05)$ entre especies y entre localidades. Al igual que en la biomasa, la especie con mayor promedio de carbono almacenado fue $G$. arborea y la localidad con mayor promedio de carbono almacenado fue Santo Domingo, y la de menor promedio fue Balzar (Cuadro 2). El promedio de carbono almacenado obtenido en esta investigación para $G$. arborea es superior a los $92.35 \mathrm{t} \mathrm{ha}^{-1}$ reportados por Jiménez (2016), en una plantación de G. arborea en Costa Rica. El promedio de carbono almacenado encontrado para $T$. ivorensis es superior a los $12.4 \mathrm{t}$ $\mathrm{ha}^{-1}$ reportados por (Magaña et al., 2004) en sistemas agroforestales con café y T. ivorensis; también fue mayor a los $71.1 \mathrm{t} \mathrm{ha}^{-1}$ obtenidos por Norgrove y Hauser (2002), en una plantación de T. ivorensis de nueve años de edad, en Camerun.

Estas diferencias, en la biomasa aérea y carbono almacenado, se puede atribuir principalmente a las densidades de las plantaciones o a las edades, pero no a los métodos de cálculo de la biomasa. En esta investigación la densidad promedio para las dos especies fue de 320 árboles ha-1 ${ }^{-1}$ En el estudio de Melo (2015) la densidad de la melina fue de $160 \mathrm{ha}^{-1}$ y 10 años de edad; en la investigación de Magaña (2004) la densidad de la terminalia fue de 250 árboles ha ${ }^{-1} \mathrm{y}$ siete años de edad; en el estudio Norgrove y Hauser (2002) la densidad fue de 93 árboles ha-1.

\section{Incremento medio anual (IMA) del volumen y fijación del carbono}

El promedio del IMA del volumen $\left(\mathrm{m}^{3} \mathrm{ha}^{-1}\right)$ presentó diferencias significativas $(\mathrm{p}<0.05)$ entre las especies $\mathrm{y}$ entre las localidades El mayor IMA del volumen promedio fue para la especie $G$. arborea y la localidad con mayor IMA del volumen fue Santo Domingo y la de menor IMA fue Balzar (Cuadro 3). El IMA del volumen para $G$. arborea obtenido en este estudio fue superior al IMA en volumen de $12.536 \mathrm{~m}^{3} / \mathrm{ha}^{-1}$ reportado por Melo (2015) en una plantación de G. arborea, de 10 años de edad, en Colombia.

El promedio del carbono fijado $\left(\mathrm{t} / \mathrm{ha}^{-1}\right)$, a los 12

Cuadro 3. Incremento medio anual en volumen $\left(\mathrm{m}^{3} / \mathrm{ha}^{-1}\right)$ de $G$. arborea $y$ T. ivorensis en tres localidades del Litoral ecuatoriano

\begin{tabular}{lcccc}
\hline Especies & \multicolumn{3}{c}{ Localidades } & Promedio \\
\cline { 2 - 4 } & Sto. Domingo & Valencia & Balzar & \\
\hline Gemelina arborea & 54,286 & 45,568 & 45,434 & $48,429 \mathrm{a}$ \\
Terminalia ivorensis & 7,138 & 6,964 & 0,590 & $4,898 \mathrm{~b}$ \\
Promedio & & & & \\
& $30,712 \mathrm{a}$ & $26,266 \mathrm{ab}$ & $23,012 \mathrm{~b}$ & \\
$\mathrm{CV}(\%)$ & & & 21.96 \\
\hline
\end{tabular}


Cuadro 4. Fijación de carbono $\left(\mathrm{t} / \mathrm{ha}^{-1}\right)$ estimado a los 12 años para G. arborea y $T$. ivorensis en tres localidades del Litoral ecuatoriano

\begin{tabular}{lcccc}
\hline Especies & \multicolumn{3}{c}{ Localidades } & Promedio \\
\cline { 2 - 4 } & Sto. Domingo & Valencia & Balzar & \\
\hline Gemelina arborea & 328.887 & 275.838 & 274.998 & $293.241 \mathrm{a}$ \\
Terminalia ivorensis & 42.827 & 41.598 & 4.615 & $29.680 \mathrm{~b}$ \\
Promedio & & & & \\
& & & & \\
CV $(\%)$ & $185.857 \mathrm{a}$ & $158.718 \mathrm{ab}$ & & 26.64 \\
\hline
\end{tabular}

años, presentó diferencias significativas $(\mathrm{p}<0.05)$ entre las especies y entre las localidades El mayor promedio del carbono fijado fue para la especie G. arborea y la localidad con mayor promedio de carbono fijado fue Santo Domingo y la de menor promedio fue Balzar (Cuadro 4). El carbono fijado, a los 12 años, para $G$. arborea obtenido en este estudio fue superior al de $129.692\left(\mathrm{t} / \mathrm{ha}^{-1}\right)$ reportado por Melo (2015) en una plantación de G. arborea, de 10 años de edad y con 320 árboles ha ${ }^{-1}$, en Colombia.

\section{Conclusiones}

$\mathrm{L}^{\mathrm{a}}$ a especie G. arborea fue la que presentó mayor cantidad de carbono almacenado y fijado, en las tres localidades estudiadas. Sin embargo, T. ivorensis es una especie con buen potencial para almacenar carbono, especialmente en la localidad de Santo Domingo. Estas dos especies forestales estudiadas pueden ser utilizadas en proyectos de reforestación, con la finalidad de almacenar carbono.

Según los resultados obtenidos en este estudio, la localidad de Santo domingo es la más recomendable para establecer plantaciones de G. arborea y T. ivorensis, ya que permite obtener mayores rendimientos en biomasa y carbono en comparación con las localidades de Valencia y Balzar.

\section{Bibliografía}

Clima Data. 2018. Clima Ecuador (en línea). Consultado nov.05 de 2018. Disponible en https://es.climate-data. org/america-del-sur/ecuador/provincia-del-guayas/ balzar-25377/

ECURED. 2018. Ecuador (en línea). Consultado nov. 05 de 2018. Disponible en https://www.ecured.cu/Ecuador.

Derek, E.; McGuinness, K.; Burrows, W. 2000. Review of allometric relationships for estimating woody biomasa for Queensland, the northern territory and western Australia. Technical report $\mathrm{N}^{0}$ 5. National Carbon accounting system. Canberra, Australia. $56 \mathrm{p}$.

FAO (Organización de las Naciones Unidas para la Agricultura y la Alimentación). 1993. Forest resources assessment 1990 tropical countries. FAO Forestry paper 112. FAO. Rome, Italy. 59 p.

Fonseca, W; Alice, F; Montero, J; Toruño, H; Leblanc, J. 2008. Acumulación de biomasa y carbono en bosques secundarios y plantaciones forestales de Vochysia guatemalensis e Hieronyma alchorneoides en el Caribe de Costa Rica

Gómez, A.; Zavala, J.; Martínez P.; López, L.; Domínguez, M.; Posada S. 2016. Carbono almacenado en la biomasa aérea de plantaciones de hule (Hevea brasiliensis Müell. Arg.) de diferentes edades. Madera y Bosques 22 (3): 49-60.

IPCC. (Intergovernmental Panel on Climate Change) 2000. Uso de la tierra, cambio de uso de la tierra y silvicultura. Informe Especial del Grupo Intergubernamental de Expertos sobre el Cambio Climático. In: Watson, R.; Verardo, D. (Eds.) Cambridge University Press. Cambridge, Reino Unido. 377 p.

IPCC (Intergovernmental Panel OnClimateChange) 2006 Directrices del IPCC de 2006 para los Inventarios Nacionales de Gases de Efecto Invernadero, Volumen 4 (Agricultura, silvicultura y otros usos de la tierra). Eds. Eggleston, S; Buendía, L; Miwa, K; Ngara, T; Tanabe, K. Hayama, J. 679 p.

IUCN (International Union for Conservation of Nature). 2018. Red List of Threatened Species (en línea). Consultado 07 nov. 2018. Disponible en http://dx.doi.org/10.2305/ IUCN.UK.1998.RLTS.T33062A9754250.en.

Jiménez, L. 2016. Evaluación de la biomasa y leña en una plantación de melina Gmelina arborea Roxb con manejo de rebrotes, Turrialba, Cartago. Tesis. Turrialba, Costa Rica. Instituto Tecnológico de Costa Rica. Escuela de Ingeniería Forestal. $41 \mathrm{p}$.

Kanninen, M.; Mery, G. 2002. Proyectos de mitigación. 
Actividades y energéticas que mitigan y actividades elegibles en el MDL. Curso Internacional de Cambio Climático en los sectores forestales y energéticos. Cartago, Costa Rica. 35 p.

Lamb, A; Ntima, O. 1971. Terminalia ivorensis. Fast Growing Timber Trees of the Lowland Tropics 5. Commonwealth Forestry Institute. Oxford, England. $72 \mathrm{p}$.

Loguercio, G; Defosse, G. 2001. Ecuaciones de biomasa aérea, factores de expansión y de reducción de la Lenga Nothofgus pumilio (popp. et Endl.) Krasser en el SO del Chubut, Argentina. Centro de Investigación y Extensión Forestal Andino-patagónico. $11 \mathrm{p}$.

Melo, O. 2015. Modelación del crecimiento, acumulación de biomasa y captura de carbono en árboles de Gmelina arborea Roxb., asociados a sistemas agroforestales y plantaciones homogéneas en Colombia. Medellín, Colombia. Universidad Nacional de Colombia. 166 p.

Montero, M; Kanninen, M. 2003. Biomasa y Carbono en plantaciones de Terminalia amazonia en la zona Sur de Costa Rica. Revista Forestal Centroamericana 39: 50-56

Montes, J. 2017. Tres nuevos registros de insectos barrenadores sobre Terminalia ivorensis A.Chev. Revista Mexicana de Ciencias Forestales 8 (43): 27-38.

Norgrove, L y Hauser, S. 2002. Measured growth and tree biomass estimates of Terminalia ivorensis in the 3 years after thinning to different stand densities in an agrisilvicultural system in southern Cameroon. Forest Ecology and Management 166 (1-3): 261-270

Rojas, F; Arias, D; Moya, R. Meza, A; Murillo, O; Arguedas, M. 2004. Manual para productores de melina Gmelina arborea en Costa Rica. San José, Costa Rica. Instituto Tecnológico de Costa Rica. 314 p.

Snowdon, P; Raison, H; Keith, K; Montagu, H; Bi, P; Ritson, P; Grieson, M; Adams, W; Burrows, D; Derek, E. 2001. Protocol for sampling tree and stand biomass. National Carbon Accounting System Technical report, no 31. Australian Greenhouse Office, Canberra, Australia. 114 p. 